\title{
ON THE DETERMINANT OF $q$-DISTANCE MATRIX OF A GRAPH
}

\author{
Hong-Hai Li ${ }^{1}$, Li SU ${ }^{2}$ AND Jing ZHANG \\ College of Mathematics and Information Science \\ Jiangxi Normal University \\ Nanchang, 330022, P.R. China \\ e-mail: suli@jxnu.edu.cn \\ lhh@mail.ustc.edu.cn \\ zhangjing3611@163.com
}

\begin{abstract}
In this note, we show how the determinant of the $q$-distance matrix $D_{q}(T)$ of a weighted directed graph $G$ can be expressed in terms of the corresponding determinants for the blocks of $G$, and thus generalize the results obtained by Graham et al. [R.L. Graham, A.J. Hoffman and H. Hosoya, On the distance matrix of a directed graph, J. Graph Theory 1 (1977) 85-88]. Further, by means of the result, we determine the determinant of the $q$-distance matrix of the graph obtained from a connected weighted graph $G$ by adding the weighted branches to $G$, and so generalize in part the results obtained by Bapat et al. [R.B. Bapat, S. Kirkland and M. Neumann, On distance matrices and Laplacians, Linear Algebra Appl. 401 (2005) 193209]. In particular, as a consequence, determinantal formulae of $q$-distance matrices for unicyclic graphs and one class of bicyclic graphs are presented.
\end{abstract}

Keywords: $q$-distance matrix, determinant, weighted graph, directed graph.

2010 Mathematics Subject Classification: 05C50,15A18.

\section{REFERENCES}

\footnotetext{
${ }^{1}$ Supported by National Natural Science Foundation of China (No.11201198 and No. 11026143), Natural Science Foundation of Jiangxi Province (No.20132BAB201013), the Sponsored Program for Cultivating Youths of Outstanding Ability in Jiangxi Normal University. Corresponding author.

${ }^{2}$ Supported by the Provincial Natural Science Foundation of Jiangxi (No.2010GZS0093), the Young Growth Foundation of Jiangxi Normal University (No. 4555).
} 
[1] R.B. Bapat, S. Kirkland and M. Neumann, On distance matrices and Laplacians, Linear Algebra Appl. 401 (2005) 193-209.

doi:10.1016/j.laa.2004.05.011

[2] R.B. Bapat, A.K. Lal and S. Pati, A q-analogue of the distance matrix of a tree, Linear Algebra Appl. 416 (2006) 799-814.

doi:10.1016/j.laa.2005.12.023

[3] R.B. Bapat and Pritha Rekhi, Inverses of q-distance matrices of a tree, Linear Algebra Appl. 431 (2009) 1932-1939.

doi:10.1016/j.laa.2009.06.032

[4] R.L. Graham and H.O. Pollak, On the addressing problem for loop switching, Bell. System Tech. J. 50 (1971) 2495-2519.

[5] R.L. Graham, A.J. Hoffman and H. Hosoya, On the distance matrix of a directed graph, J. Graph Theory 1 (1977) 85-88. doi:10.1002/jgt.3190010116

[6] S.G. Guo, The spectral radius of unicyclic and bicyclic graphs with $n$ vertices and $k$ pendant vertices, Linear Algebra Appl. 408 (2005) 78-85. doi:10.1016/j.laa.2005.05.022

[7] S. Sivasubramanian, A q-analogue of Graham, Hoffman and Hosoya's result, Electron. J. Combin. 17 (2010) \#21.

[8] P. Lancaster, Theory of Matrices (Academic Press, NY, 1969).

[9] W. Yan, Y.-N. Yeh, The determinants of q-distance matrices of trees and two quantities relating to permutations, Adv. in Appl. Math. 39 (2007) 311-321.

doi:10.1016/j.aam.2006.04.002

Received 7 August 2012

Revised 13 December 2012

Accepted 13 December 2012 